\title{
EFFECTS OF QUADRICEPS FATIGUE ON KNEE JOINT KINETICS DURING SLIP INDUCED FALLS
}

\author{
Prakriti Parijat $^{1}$ and Thurmon Lockhart ${ }^{2}$ \\ ${ }^{1}$ School of Biomedical Engineering and Sciences, Virginia Tech, ${ }^{2}$ Industrial Systems and Engineering, Virginia Tech \\ Corresponding author email: pparijat@vt.edu
}

\section{INTRODUCTION}

Occupationally induced localized muscular fatigue (LMF) has been recently identified as one of the major intrinsic factors contributing to slip and fall accidents [1]. Although there has been a reduction of heavy work due to growing technological advances, some occupations such as construction, and forestry still demand intense physical work. Literature indicates that a third of the U.S. workforce exerts significant physical strength as part of their jobs, and experience fatigue at their work places [2]. While epidemiological studies link the incidence of slip-induced falls with LMF, the mechanism associated with fatigue and its relationship with slip-induced fall accidents still remains unclear. The purpose of the present study was to examine how lower extremity fatigue (quadriceps femoris) alters joint moment and increases slip severity.

\section{METHODS}

Sixteen healthy young participants were recruited to walk across an unexpected slippery floor in two different sessions (Fatigue (F) and No fatigue (NF)) in a span of one week. Each of these sessions comprised of normal walking and slip trials. Bilateral quadriceps fatigue was induced using isokinetic exertions of the knee during the fatigue trial. Fatigue inducement procedure were similar to previous literature [3]. Kinematic and kinetic data were collected using a three-dimensional video analysis system and force plates during both sessions. An inverse dynamics approach was adopted for the joint moment determination [4]. The required coefficient of friction (RCOF), sagittal peak knee joint moment and frequency of falls important in assessing slip severity were compared for the two different sessions to evaluate the effects of fatigue. A repeated measure one-way analysis of variance (ANOVA) was employed to predict statistical significance.

\section{RESULTS AND DISCUSSION}

The results indicated that RCOF was higher in normal walking trials in F session (Table 1). During normal walking trials in both NF and F sessions, consistent alternate flexor and extensor joint moment patterns were observed in all participants. Three distinctive peaks (P1-extensor, P2-flexor and P3-extensor) were observed and analysed. P3 was significantly lower in $\mathrm{F}$ session. The knee joint moment profile in Figure 1 is an ensemble average of all the participants who had a reactive recovery during slip trials in $F$ and NF sessions. Peak joint moment was extensor

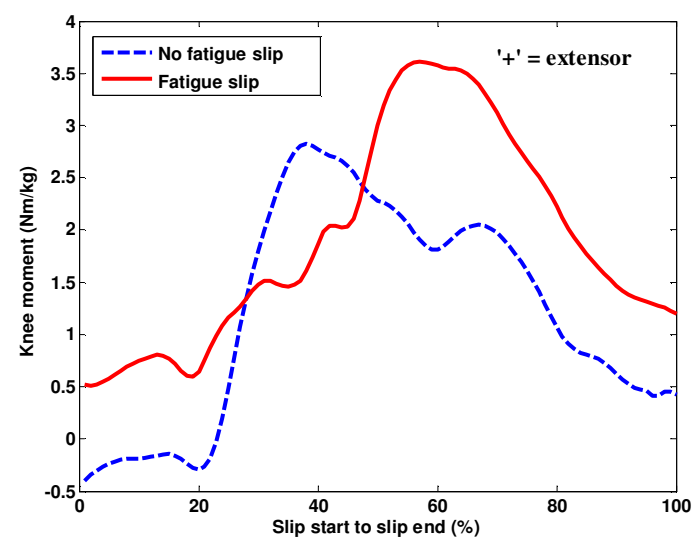

Figure 1. Average knee joint moment profile during reactive recovery in no fatigue and fatigue session (slip trial).

dominant and significantly higher during the slip-recovery in $F$ session (Table 1). There were four falls in the F session and one fall in the NF session. Higher RCOF has been related to increase in the slip propensity and this could be due to the increased ground reaction forces due to fatigue. It was interesting to observe a higher knee joint moment to recover from a slip in F session, which was contradictory to what was expected. Even though participants recovered, the higher demands imposed on the fatigued knee might lead to overexertion injury. A large variability in the slip data was observed which might be due to difference in fatigue levels Overall, there were more falls in the F session and this may be linked with lack of sufficient joint moment production.

\section{CONCLUSIONS}

The present study concluded that LMF of the knee can be considered a potential risk factor for slip induced falls. However, further analysis is required to examine factors such as joint power, joint moment reaction time to completely explain effects of fatigue on the knee joint behavior during slip induced falls/recovery.

\section{REFERENCES}

1. Hsiao H, et al. Ergonomics 44, 537-561, 2001.

2. Swaen G, et al.. Occup Environ Med 60, (SuppI):i82-i92, 2003.

3. Yaggie J, et al., Arch of Phys Med and Rehab 83, 224-228, 2002.

4. Liu J, et al.,Gait \&Posture 23, 480-485, 2006.

Table 1: Mean and standard deviation of the peak knee moment (kneemom $\left.{ }_{\text {peak }}\right)$ and required coefficient of friction (RCOF) during the no fatigue and fatigue sessions, $* \mathrm{p}<0.05$

\begin{tabular}{|c|c|c|c|c|c|}
\hline \multirow[t]{2}{*}{ Condition } & \multirow{2}{*}{$\begin{array}{c}\text { RCOF- normal } \\
\text { walking trial }\end{array}$} & \multicolumn{3}{|c|}{ Kneemoment $_{\text {peak }}$-normal walking trial $(\mathrm{Nm} / \mathrm{kg})$} & \multirow{2}{*}{$\begin{array}{l}\text { Kneemoment }_{\text {peak }} \\
\text {-slip trial }(\mathrm{Nm} / \mathrm{kg})\end{array}$} \\
\hline & & P1 & P2 & P3 & \\
\hline No Fatigue & $0.15(0.007)^{*}$ & $0.45(0.19)$ & $0.15(0.29)$ & $0.45(0.26)^{*}$ & $2.44(0.97)^{*}$ \\
\hline Fatigue & $0.18(0.005)$ & $0.23(0.22)$ & $0.04(0.07)$ & $0.23(0.20)$ & $3.92(1.83)$ \\
\hline
\end{tabular}

